\title{
Threonine Measurement
}

National Cancer Institute

\section{Source}

National Cancer Institute. Threonine Measurement. NCI Thesaurus. Code C122156.

The determination of the amount of threonine present in a sample. 